\title{
Etude in situ de la réinstallation d'un peuplement sciaphile de mode battu après sa destruction expérimentale, en Méditerranée
}

\author{
C.-F. Boudouresque \\ Laboratoire de Biologie végétale marine, \\ $U E R$ des Sciences de la Mer et de l'Environnement; \\ Marseille, France
}

\begin{abstract}
In situ studies on the reconstruction of a shady and wave-lashed marine population after experimental destruction in the Mediterranean. The structure (internal organization) of the biocoenosis of the shady and wave-lashed bottoms in the western Mediterranean Sea is very heterogeneous. Little is known concerning the causes of this heterogeneity. For analyzing the composition of the vegetation, the survey method of BRAUN-BLANQUET (1964) was used. Reconstruction of the biocoenosis on cleaned rocky surfaces follows a definite pattern as a function of time. After 4 months, the rocky surface is covered with the crustaceous alga Dermatolithon hapalidioides. After 6 months, various dendristic and fruticose algae settle on $D$. hapalidioides, mainly Pseudochlorodesmis furcellata, Petroglossum nicaeense and Myriogramme minuta. After 1 year, the whole area is occupied by $P$. nicaeense, Plocamium vulgare and Valonia utricularis; the undergrowth consists of $D$. hapalidioides which becomes slowly superseded by fruticose algae. This sociological progression shows that: (1) The phases during reconstruction of the settlement are equivalent to the structural elements of the initial settlement; (2) perhaps the structural organization results from a duronological sequence of different phases; (3) reconstruction of the initial settlement after experimental destruction is very rapid.
\end{abstract}

\section{INTRODUCTION}

Les biotopes sciaphiles superficiels de mode battu sont occupés, en Méditerranée occidentale, par une biocoenose originale, la biocoenose de la Roche Sciaphile en mode Battu (RSB). La faune de cette biocoenose ne semble pas très originale (BeLLAN-SANTINI 1962, 1969); par contre, sa flore est plus originale: une quarantaine d'espèces d'algues peuvent en effet être considérées comme des caractéristiques exclusives ou preférentielles de la biocoenose de la roche sciaphile en mode battu (RSB) (BoudouResque 1969, 1971a, 1971b, Boudouresque \& Passelatgue 1972).

La biocoenose de la roche sciaphile en mode battu est represésentée par une multitude de faciès\% qui n'occupent que rarement des surfaces importantes: à quelques

* Une biocoenose présente un faciès lorsque la prédominance locale de certains facteurs écologiques entraine l'exubérance d'une ou d'un petit nombre d'espèces (que ces espèces soient caractéristiques ou non de la biocoenose), sans que pour cela la composition qualitative de la biocoenose en soit modifiée (PÉRÈs \& PICARD 1964). 
mètres de distance, ils se remplacent les uns les autres, donnant ainsi à la biocoenose un aspect varié (Boudouresque 1971d).

Au sein d'un faciès donné, les variations saisonnières paraissent peu importantes, au moins dans la région marseillaise (Boudouresque \& Passelaigue 1972); la stratification et la structure élémentaires sont, par contre, presque toujours complexes: des espèces ou des groupes d'espèces forment des taches, des éléments au sens de Gounot (1969), imbriqués en une mosaïque assez régulière (Boudouresque \& Lück 1972).

L'étude structurale (Boudouresque \& Lück 1972) a démontré la forte hétérogénéité structurale de la RSB; les éléments mis en évidence ne sont pas le rappel, à une plus petite échelle, des nombreux faciès de la RSB. A la suite de l'étude structurale, l'hypothèse a donc été émise que l'aspect en mosaïque de la biocoenose de la roche sciaphile en mode battu (RSB) pourrait être lié aux phases d'une évolution cyclique, comparable par exemple à celle des tourbières dans le domaine terrestre. Seule une étude in situ de la régénération de la RSB après sa destruction expérimentale pouvait confirmer ou infirmer cette hypothèse.

Il est par ailleurs évident qu'une telle étude est indispensable pour tester la valeur réelle d'une biocoenose-type, c'est-à-dire son équilibre avec le milieu, sa reproductibilité, ou au contraire son déséquilibre ou son caractère aléatoire. Nous ne ferons pas ici l'historique des nombreux travaux consacrés aux régénérations de peuplements benthiques après dénudation expérimentale ou sur substrat solide vierge, renvoyant pour cela à la bibliographie que donne Huvé $(1969,1970)$.

\section{MATERIEL ET METHODES}

L'expérimentation a été poursuivie parallèlement sur la face Nord de l'île Riou, près de Marseille, au sein d'un faciès type à Petroglossum nicaeense et Plocamium vulgare, et au Cap du Troc, au Sud-Est de Banyuls (Pyrénées-Orientales), au niveau d'un faciès à Corallina mediterranea. Les conclusions étant concordantes pour l'essentiel, seule la première de ces deux expériences sera détaillée ici.

Comme il a été dit plus haut, la biocoenose-type RSB se présente sous un grand nombre de faciès, occupant parfois une surface assez faible. Au niveau des biotopes sciaphiles superficiels les conditions de milieu changent souvent très vite d'un point à un autre. C'est pour ces raisons, et afin de se placer dans des conditions d'expérience aussi rigoureuses que possible, que la même surface a éte suivie d'année en année, ou de mois en mois (au détriment évidemment du nombre de prélèvements).

La biocoenose dont la régénération allait être étudiée a été au préalable analysée soigneusement grâce à quatre relevés phytosociologiques (relevés $\mathrm{N}^{\circ} 225,273,321$ et 327) échelonnés tout au long de l'année, ce qui permet d'obtenir une moyenne annuelle prise comme référence (peuplement de référence, dernière colonne de Tableau 1).

Six relevés (relevés $\mathrm{N}^{\circ} 357,354,355,336,333,274$ ) permettent de suivre l'évolution de la régénération après $1,2,3,4,6$ et 12 mois; pour tous ces relevés, la dénudation a été effectuée entre les mois d'octobre et de janvier, c'est à dire à l'automne ou au début de l'hiver (sauf́ le relevé $\mathrm{N}^{\circ} 336$ : juillet).

Il n'est pas possible d'exposer ici en détail la méthodologie utilisée pour la récolte 
Tableau 1

Régénération d'une biocoenose de la roche sciaphile en mode battu, et peuplement de référence (moyenne de 4 relevés). Dans la dernière colonne, le recouvrement moyen est exprimé en pourcentage. Dans les autres colonnes: coefficients de recouvrement et de sociabilité, séparés par un point

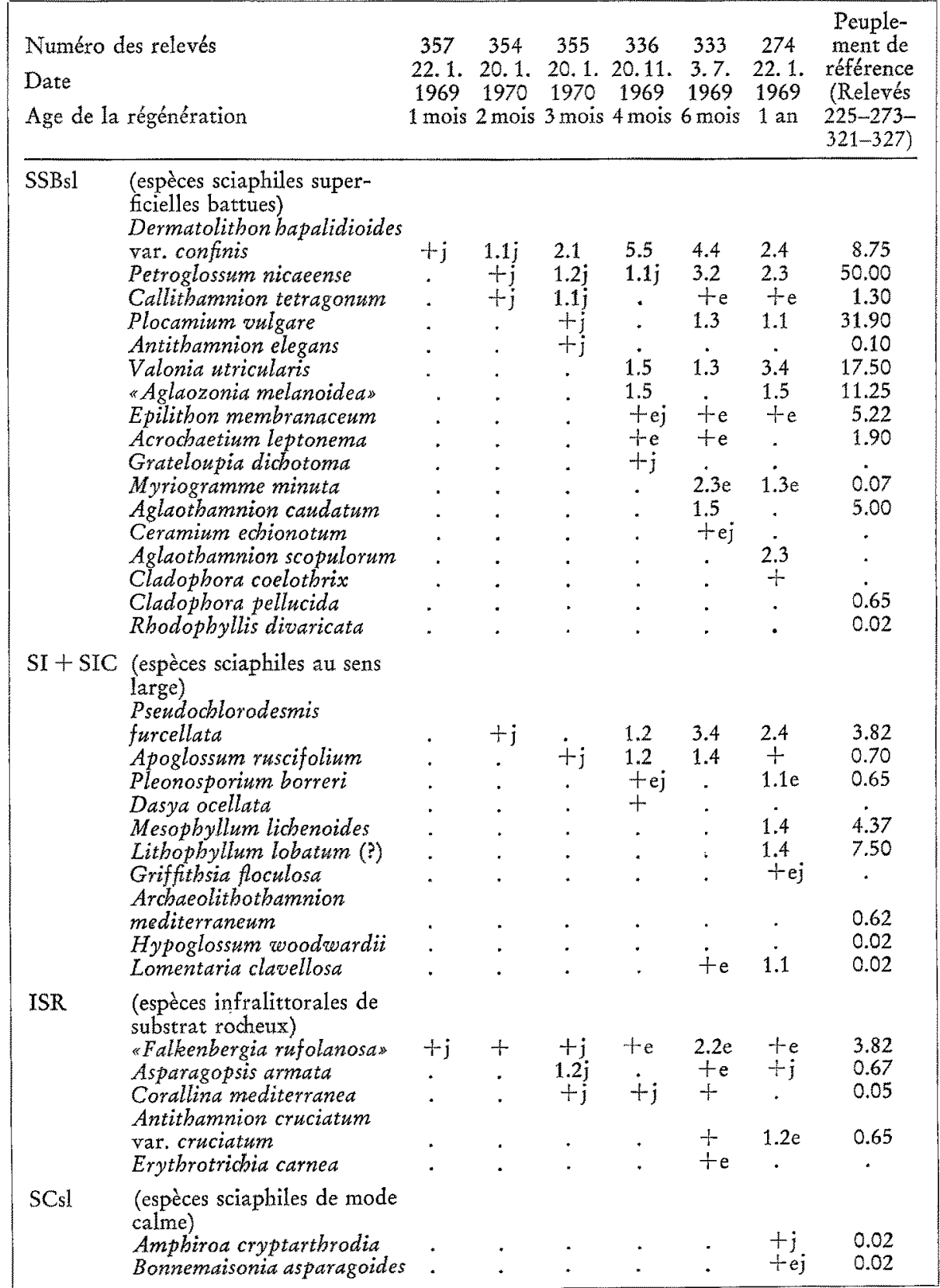


Tableau 1 (suite)

\begin{tabular}{|c|c|c|c|c|c|c|c|c|}
\hline \multicolumn{2}{|c|}{$\begin{array}{l}\text { Numéro des relevés } \\
\text { Date } \\
\text { Age de la régénération }\end{array}$} & $\begin{array}{c}357 \\
22.1 . \\
1969 \\
1 \text { mois }\end{array}$ & $\begin{array}{c}354 \\
20.1 \\
1970 \\
2 \text { mois }\end{array}$ & $\begin{array}{c}355 \\
20.1 . \\
1970 \\
3 \text { mois }\end{array}$ & $\begin{array}{c}336 \\
20.11 \\
1969 \\
4 \text { mois }\end{array}$ & $\begin{array}{c}333 \\
3.7 \\
1969 \\
6 \text { mois }\end{array}$ & $\begin{array}{l}274 \\
22.1 . \\
1969 \\
1 \text { an }\end{array}$ & $\begin{array}{c}\text { Peuple- } \\
\text { ment de } \\
\text { référence } \\
\text { (Relevés } \\
225-273- \\
321-327 \text { ) }\end{array}$ \\
\hline \multirow{8}{*}{ APsl } & Peyssonnelia squamaria & . & . & $\therefore$ & . & . & $\cdot$ & 0.02 \\
\hline & Antithamnion plumula & . & . & . & . & & . & 0.02 \\
\hline & Giffordia sandriana & . & . & . & . & $+\mathrm{e}$ & . & . \\
\hline & $\begin{array}{l}\text { (espèces photophiles } \\
\text { infralittorales) }\end{array}$ & & & & & & & \\
\hline & $\begin{array}{l}\text { Bryopsis duplex } \\
\text { Dilophus fasciola var. }\end{array}$ & . & $+j$ & $+\mathrm{j}$ & 2.3 & 1.4 & $1.2 \mathrm{j}$ & 0.05 \\
\hline & $\begin{array}{l}\text { repens } \\
\text { Laurencia pinnatifida }\end{array}$ & : & : & $\begin{array}{l}+j \\
+j\end{array}$ & $+j$ & . & : & $\begin{array}{l}0.02 \\
0.00\end{array}$ \\
\hline & Ceramium rubrum & : & : & . & $\dot{.}$ & $\dot{+} \mathrm{ej}$ & . & .00 \\
\hline & Sphacelaria cirrosa & * & . & . & . & . & $+\mathrm{j}$ & . \\
\hline \multirow[t]{4}{*}{ RMsl } & (espèces médiolittorales) & & & & & & & \\
\hline & Callitbamnion tetragonum & : & +1 & : & $\begin{array}{l}+e j \\
+e j\end{array}$ & : & : & : \\
\hline & Chaetomorpha capillaris & & & & & & & \\
\hline & var. crispa & . & . & . & . & . & . & 0.02 \\
\hline \multicolumn{9}{|c|}{$\begin{array}{l}\text { SSP-LRE (espèces sans signification } \\
\text { précisée, à large répartition } \\
\text { écologique et diverses) }\end{array}$} \\
\hline & Cyanophytes indéterminés & 2.1 & 3.1 & 2.1 & + & te & 2.1 & 5.62 \\
\hline & Diatomées indéterminées & 2.1 & 4.1 & 3.3 & te & te & $+\mathrm{e}$ & 1.30 \\
\hline & Licmophora sp. & . & 1.1 & $1,2 \mathrm{e}$ & $+e$ & $+\mathrm{e}$ & . & 0.65 \\
\hline & Cladophora sp. & . & $+j$ & I & & . & . & . \\
\hline & Ulva sp. & . & . & $+\mathrm{j}$ & $1.2 \mathrm{j}$ & & . & . \\
\hline & Erythrotricbia sp. & . & . & $+e j$ & . & te & . & . \\
\hline & Bryopsis adriatica (?) & . & . & 1.1 & . & . & . & . \\
\hline & Cutleria adspersa & 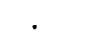 & . & $+\mathrm{j}$ & & . & & \\
\hline & Polysiphonia sp. & . & . & . & + & + & $+e j$ & 0.67 \\
\hline & Goniotrichum cornu-cervi & $\cdot$ & . & . & $+e j$ & $1.2 \mathrm{e}$ & . & 0.02 \\
\hline & Erytbrocladia subintegra & . & . & . & te & . & . & . \\
\hline & Cladophora sp. & $\cdot$ & . & . & $+j$ & 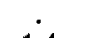 & . & \\
\hline & Lithothamnium lenormandii & . & . & . & . & 1.4 & & 2.50 \\
\hline & Dasya corymbifera & . & . & . & 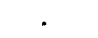 & + & $+e j$ & . \\
\hline & Callitbamniella tingitana &  & . & . & & . & $+\mathrm{e}$ & \\
\hline & Phormidium sp. & . & . & . & . & . & . & 0.02 \\
\hline & Derbesia tenuisssima & . & ${ }^{\circ}$ & . & 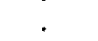 & . & . & 0.62 \\
\hline & Entocladia viridis & . & : & : & : & . & : & 0.02 \\
\hline & Ectocarpus confervoides &  & . & . & . & . & . & 0.02 \\
\hline & Compsonema (?) sp. & $\cdot$ & $\cdot$ & . & 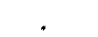 & . & . & 0.02 \\
\hline & Acrocbaetium sp. & . & . & . & . & . & . & 0.02 \\
\hline & Mélobésiées indéterminées & . & • & . & . & • & . & 1.28 \\
\hline & Dermatolithon pustulatum & 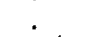 & . & . & 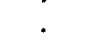 & . & . & 0.02 \\
\hline
\end{tabular}

et l'analyse des relevés: nous renvoyons donc à Boudouresque (1971c); nous nous contenterons de rappeler quelques principes et définitions:

(1) Chaque relevé a consisté en un grattage complet de la surface étudiée $(10 \times 15 \mathrm{~cm})$; la roche a été cassée sur 5 à $10 \mathrm{~mm}$ d'épaisseur, afin que le calcairc urgonien (substratum) soit réellement abiotique après dénudation. 
(2) Lors du tri des relevés en laboratoire, la liste des espèces présentes est établie, et deux coefficients sont attribués à chaque espèce, selon la méthode de BRAUN-BLANQUET $(1959,1964)$; le premier coefficient est le recouvrement, noté du + à 5 ; le second est la sociabilité, noteé de 1 à 5 ; un point sépare ces deux coefficients (Tableau 1, colonnes 1 à $6 ; \mathrm{e}=$ epiphyte; $\mathrm{j}=$ juvénile).

(3) La moyenne des recouvrements d'une espèce portant sur plusieurs relevés (recouvrement moyen global $=\mathrm{RMG}$ ) est exprimée en pourcentage (dernière colonne $\mathrm{du}$ Tableau 1).

(4) Dans le Tableau 1, les espèces sont réunies en un certain nombre de groupes et de supergroupes écologiques, en fonction de leurs affinités écologiques et sociologiques en Méditerranée occidentale. Pour alléger la texte, ces groupes et supergroupes écologiques sont désignés par des initiales: APsI = espèces photophiles infralittorales; $\mathrm{ISR}=$ espèces infralittorales de substrat rocheux; $\mathrm{RMsl}=$ espèces médiolittorales SCsI = espèces sciaphiles de mode relativement calme; SI + SIC = espèces sciaphiles; au sens large; SSBsl = espèces sciaphiles superficielles de mode battu; SSP-LRE = espèces sans signification précisée, à large répartition écologique, et diverses.

(5) La densité de reproduction, qui a été définie récemment pour permettre de chiffrer l'importance des phénomènes reproductifs au niveau d'un peuplement (BouDOURESQUE 1971c), peut prendre des valeurs comprises entre 0 et 10.

(6) L'indice de diversité de Margalef mesure, dans un peuplement, la quantité totale d'information résultant de la différenciation en espèces.

(7) Le coefficient de similitude de KulczYnsKr (1927) permet de comparer globalement des relevés:

$$
S_{k}=\frac{2 . \sum_{i=1}^{n} \inf \left(R_{i p}, R_{i q}\right)}{\sum_{i=1}^{n} R_{i p}+\sum_{i=1}^{n} R_{i q}}
$$

où inf $\left(R_{i p}, R_{i q}\right)$ représente la plus petite des deux valeurs $R_{i p}$ et $R_{i q}$ du recouvrement de l'espèce $\mathrm{i}$ dans les relevés $\mathrm{p}$ et $\mathrm{q}$.

(8) Des pesées espèce par espèce (poids humide égoutté non décalcifié) ont été effectuées pour tous les relevés.

\section{ETUDE DES PARAMETRES DU PEUPLEMENT AU COURS DE SA REGENERATION}

L'étude d'un certain nombre de paramètres du peuplement au cours de sa régénération montre qu'ils tendent, en rêgle générale, à retrouver plus ou moins vite la valeur qu'ils avaient dans le peuplement de référence.

Le nombre d'espèces par relevé (Fig. 1a) qui est de 27.5 dans le peuplement de référence, est déjà de 20 au troisième mois; à partir du 6ème mois, il devient même supérieur à 27.5: des espèces que l'on rencontre habituellement dans d'autres biocoenoses, telles Callithamniella tingitana et Laurencia pinnatifida, s'installent; elles sont éliminées par la suite. 
En revanche, la croissance de la biomasse est bien plus lente; après un an, le poids humide égoutté dépasse de peu $1 \mathrm{~kg} / \mathrm{m}^{2}\left(1055 \mathrm{~g} / \mathrm{m}^{2}\right)$; c'est à partir du 4ème mois que la biomasse de peuplement régénéré devient brusquement appréciable (Fig. 1b); ce qui
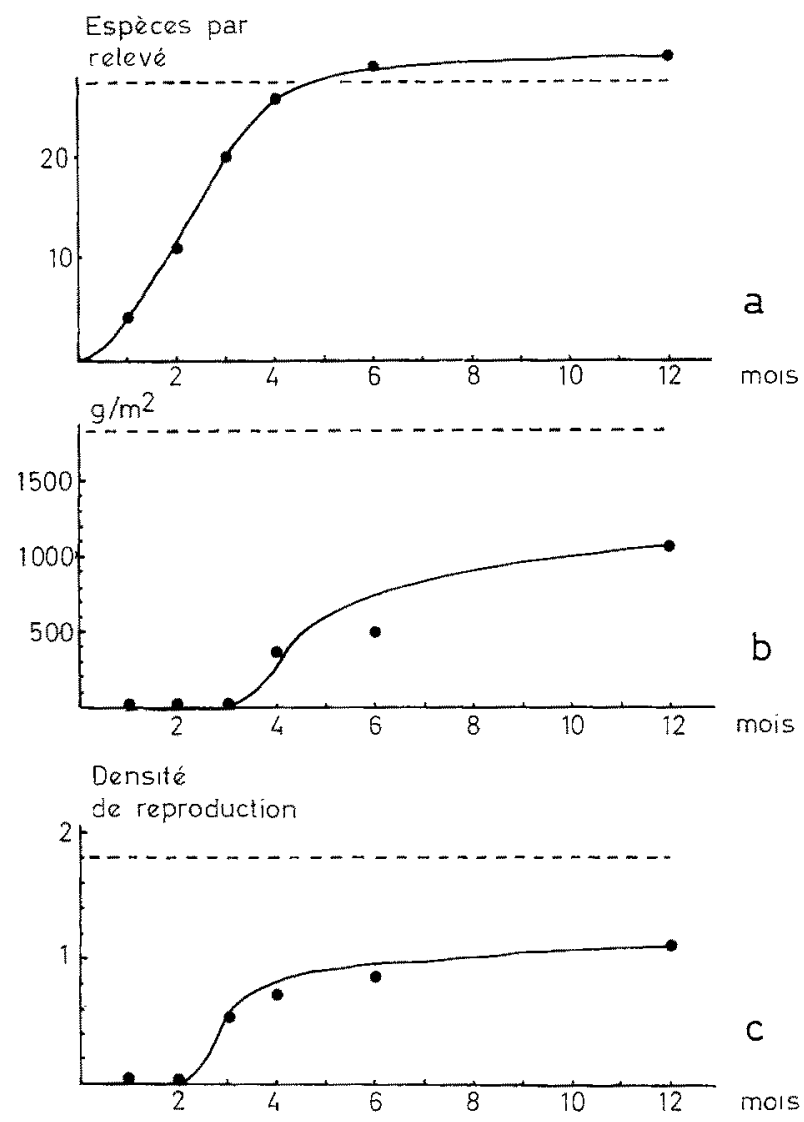

Fig. 1: a Evolution du nombre d'espèces par relevé au cours de la régénération du peuplement. $b$ Evolution de la biomasse au cours de la régénération du peuplement. $c$ Evolution de la densité de reproduction au cours de la régénération du peuplement. - . = Moyenne pour le peuplement de référence

apparaît encore mieux en coordonnées logarithmiques (Fig. 2): en effet, la courbe de la figure $1 \mathrm{~b}$ peut laisser croire que l'accroissement de la biomasse commence entre le 3ème et le 4ème mois alors qu'en réalité (Fig. 2) il commence dès le ler mois. C'est un saut quantitatif brutal qui s'est produit entre le 3ème et le 4ème mois.

C'est à partir du 3ème mois que la densité de reproduction prend une valeur appréciable (Fig. 1c); à partir de ce moment-là, elle augmente lentement, mais, au 12ème mois, elle est encore nettement inférieure à sa valeur de référence (1.10 au lieu de 1.78). 
Tableau 2

Evolution de quelques paramètres au cours de la régénération

\begin{tabular}{|lccc|}
\hline Relevés $N^{\circ}$ & $\begin{array}{c}\text { Nombre } \\
\text { d'espèces/relevé }\end{array}$ & $\begin{array}{c}\text { Densité de } \\
\text { reproduction }\end{array}$ & $\begin{array}{c}\text { Indice de } \\
\text { diversité }\end{array}$ \\
\hline $357 \quad($ I mois $)$ & 4.0 & 0.00 & 1.075 \\
354 ( 2 mois) & 11.0 & 0.00 & 1.301 \\
355 ( 3 mois $)$ & 20.0 & 0.54 & 2.202 \\
336 ( 4 mois $)$ & 26.0 & 0.72 & 1.640 \\
333 (6 mois) & 29.0 & 0.84 & 2.712 \\
$274 \quad(12$ mois $)$ & 30.0 & 1.10 & 3.307 \\
\hline Peuplement de & & & 3.078 \\
référence & 27.5 & 1.78 & \\
\hline
\end{tabular}

Enfin, l'indice de diversité de Margalef (Tableau 2) augmente rapidement et prend au bout d'un an une valeur supérieure à sa valeur de référence. Ceci n'a rien d'étonnant; l'occupation plus ou moins anarchique de la «place vide» par un grand nombre d'espèces tend à créer une quantité d'information supérieure à son niveau habituel. L'apparition d'un milieu endogène, véritable circuit ufeed-back», corrigera ultérieurement une seconde fois cette déviation.

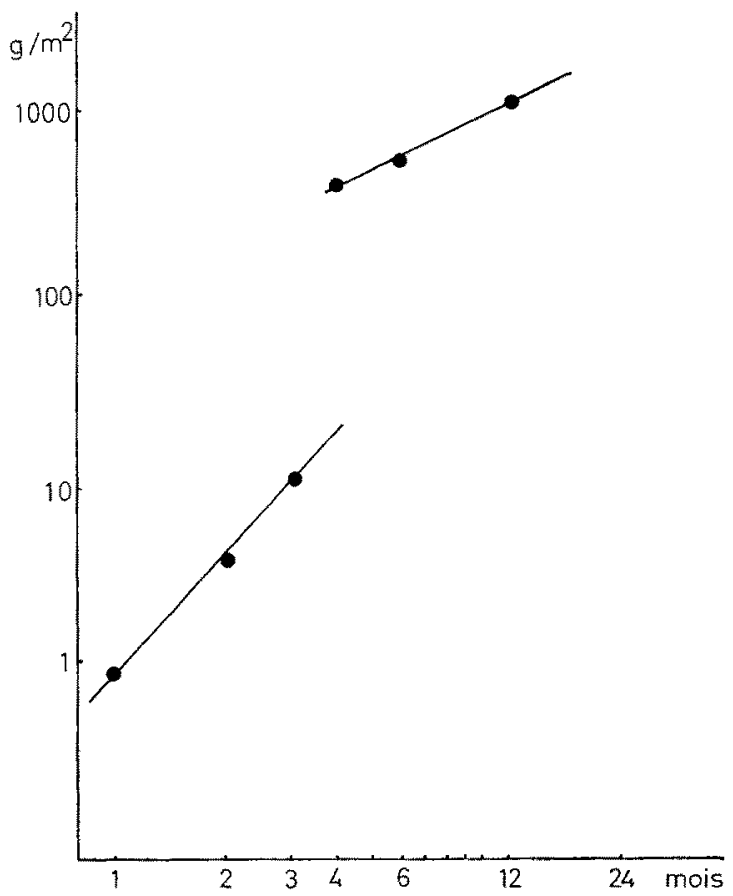

Fig. 2: Evolution de la biomasse (en coordonnées logarithmiques) au cours de la régénération du peuplement 


\section{ETUDE DES AFFINITES BIOCOENOTIQUES DU PEUPLEMENT AU COURS DE SA REGENERATION}

D'un point de vue biocoenotique, il apparaît que le supergroupe écologique SSBsl, qui caractérise la biocoenose-type RSB, se met en place très vite, tant pour le recouvrement (Tableau 1) que pour la biomasse et la dominance en fonction de la bio-

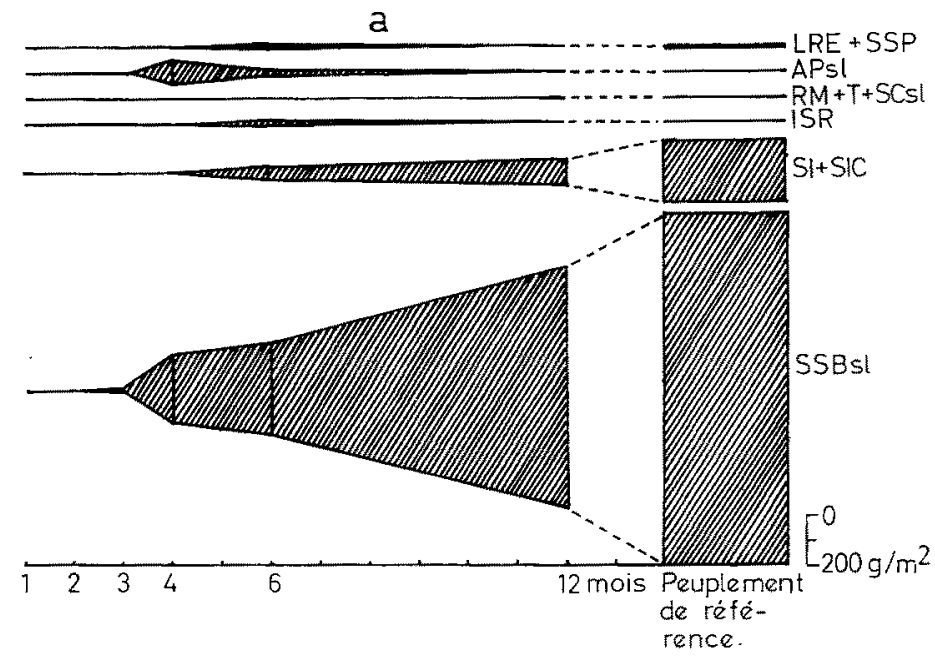

$\mathrm{b}$



Fig. 3: Evolution de la biomasse des différents groupes et supergroupes écologiques $(a)$ et de leur Dominance en fonction de la biomasse $(b)$ au cours de la régénération du peuplement

masse (Fig. 3); à partir du 3ème mois, sa dominance est en effect voisine de celle qu'il possède dans le peuplement de référence. On aboutit aux mêmes conclusions en considérant le nombre d'espèces par relevé appartenant au supergroupe SSBsl (Tableau 3).

Ce qu'il convient surtout de remarquer, c'est la tentative d'installation, dès le deuxième mois, de quelques espèces, (surtout Bryopsis duplex) du supergroupe photophile infralittoral (APsl). Ce supergroupe (en noir sur la Fig. 3) a une réelle importance au 4ème mois, où sa dominance en fonction de la biomasse atteint $23.6 \%$; puis, il est 
Tableau 3

Evolution du nombre d'espèces SSBsl et du coefficient de similitude avec le peuplement de référence, au cours de la régénération

\begin{tabular}{|c|c|c|}
\hline Relevés No & $\begin{array}{l}\text { Espèces SSBsl } \\
\text { par relevé }\end{array}$ & $\begin{array}{l}\text { Coefficient similitude } \\
\text { KuLCZYNSKI }\end{array}$ \\
\hline 357 ( 1 mois) & 1.0 & 0.071 \\
\hline 354 ( 2 mois $)$ & 3.0 & 0.076 \\
\hline 355 ( 3 mois $)$ & 6.0 & 0.177 \\
\hline 336 (4 mois) & 8.0 & 0.151 \\
\hline 333 ( 6 mois) & 10.0 & 0.362 \\
\hline 274 (12 mois) & 11.0 & 0.401 \\
\hline $\begin{array}{l}\text { Peuplement de } \\
\text { référence (moyenne } \\
\text { sur } 4 \text { relevés) }\end{array}$ & 11.5 & $\# 0.700$ \\
\hline
\end{tabular}

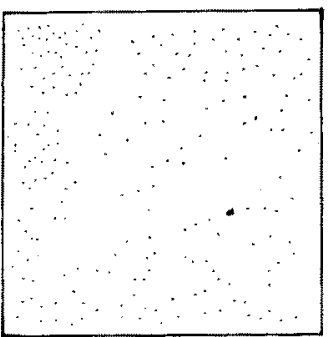

1 mois

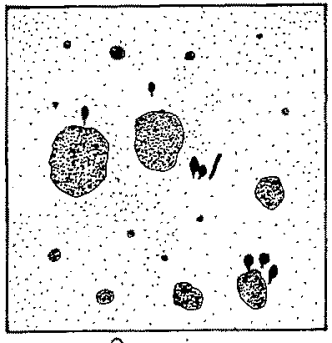

3 mois

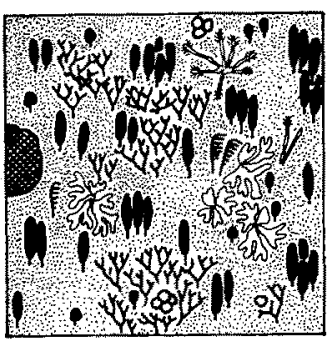

6 mois

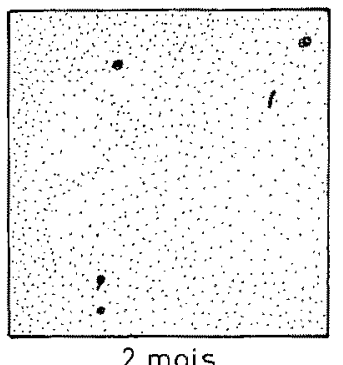

2 mois
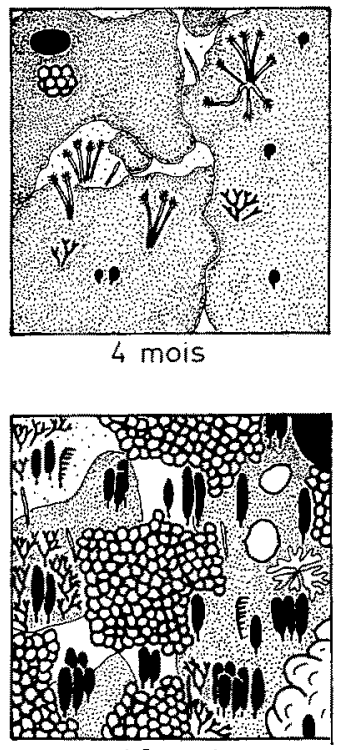

12 mois
Diatomées et

Cyanophycées

(1) Dermatolithon

hapalidioides

Lithophyllum lobatum (2) Mesophyllum liW1 Petroglossum ni-

Flocamium vulgare

Lithothamnion lenormandii

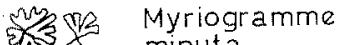
minuta

Valonia utricula. ris

Pseudochlorodesmis furcellata

\section{Bryopsis duplex}

Aglaozonia mela noidea

Fig. 4: Aspect semi-shématique du peuplement régénéré après $1,2,3,4,6$ et 12 mois 
progressivement éliminé: $4.5 \%$ au 6ème mois, $0.6 \%$ au 12 ème mois, et $0.01 \%$ dans le peuplement de référence. Il s'agit donc d'un véritable phénomène de rejer: un supergroupe étranger au biotope, et quì n'a pu s'y introduire qu'à la faveur d'une diminution provisoire de la pression de concurrence (modification du milieu endogène), est éliminé en quelques mois.

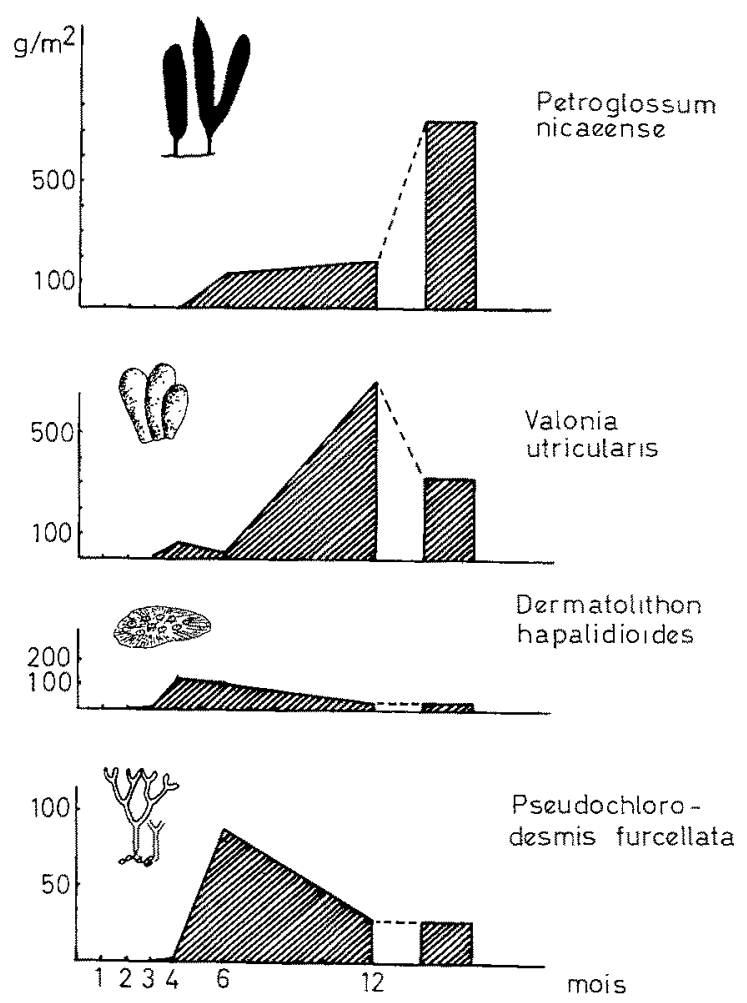

Fig. 5: Evolution de la biomasse de quelques espèces, au cours de la régénération du peuplement

Le cas de Bryopsis duplex est particulièrement intéressant; cette espèce est pratiquement absente du peuplement de référence, et cela quelle que soit l'époque de l'année (il s'agit en effet d'une espèce à optimum estival). Son installation dans un biotope sciaphile superficiel battu, et la vitalité normale qu'elle y connait, montrent qu'elle peut très bien y vivre, mais qu'elle n'y est pas compétitive.

Cet exemple montre bien le danger qu'il y a à confondre les exigences écologiques d'une espèce (telles que l'on peut les déduire d'un nombre élevé d'observations ainsi que de cultures dans des conditions aussi proches que possible de la réalité supposée) avec sa signification biocoenotique (synthèse statistique de ses réactions aux innombrables facteurs écologiques qu'elle peut rencontren). En particulier, l'observation instantanée d'une espèce hors de son biotope habituel (observation intéressante en ellemême), même si sa vitalité et sa reproduction paraissent normales, ne peut suffire à réfuter une interprétation biocoenotique fortement étayée. 
Le coefficient de similitude de KuLCzYNSKI entre le peuplement régénéré et le peuplement de référence traduit très bien le retour de l'ensemble de peuplement (quelle que soit la signification écologique des espèces qui le constituent) en direction du peuplement de référence, qui apparaît donc comme un état d'équilibre. Il existe d'ailleurs une bonne corrélation positive entre le coefficient de KuLCzYNSKI et l'indice de Margalef.

\section{PHASES DE LA REGENERATION}

L'ensemble des peuplements régénérés de la face Nord de l'île Riou, ainsi que le stade initial ( 0 ) et le peuplement de référence ( $R$ ), sont schématisés (Fig. 6) dans un espace paramétrique à 2 dimensions. Les différents stades de la régénération sont maté-

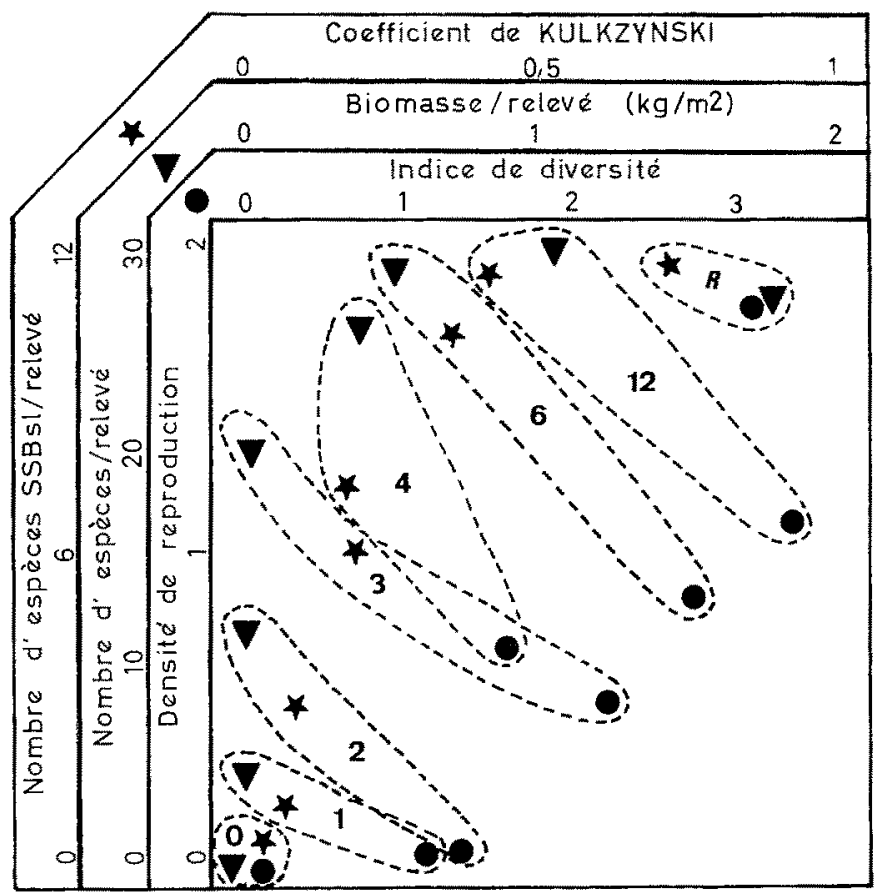

Fig. 6: Représentation des stades successifs de la régénération du peuplement dans un espace paramétrique à deux dimensions; chaque signe (point, triangle, étoile) se situe à l'intersection des valeurs des deux paramètres auxquels il correspond

rialisés par un trait pointillé. Ce schéma synthétique traduit, mieux que toute digression, le retour d'ensemble du peuplement, de ses paramètres, de ses caractères, de ses affinités (avec des vitesses diverses) vers un état d'équilibre constitué par le peuplement. de référence.

Les deux premiers mois de la régénération correspondent à une phase de latence (Fig. 4); diatomées et cyanophytes se partagent le substrat disponible. Entre le 4ème 
et le 6ème mois, une algue calcaire encroûtante, Dermatolitbon bapalidioides var. confinis, envahit la presque totalité de la roche, jusqu'à avoir un recouvrement voisin de $100 \%$ (Fig. 4). Nous pouvons parler d'une «phase à Dermatolithon hapalidioides».

C'est au 6ème mois que Pseudochlorodesmis furcellata est à son optimum; il commence à constituer, avec Petroglossum nicaense, Plocamium vulgare, Myriogramme minuta, une strate élevée; si la distribution de Pseudocblorodesmis furcellata est nettement contagieuse, celle de Petroglossum nicaeense et de Plocamitum vulgare semble plutôt aléatoire.

Au 12ème mois (Fig. 4), la strate encroûtante formée par Dermatolithon hapalidioides est en grande partie recouverte par Valonia utricularis (espèce présente toute l'année, mais présentant néanmoins un optimum hivernal et automnal), ainsi que par le développement de la strate dressée (Petroglossum nicaeense, Plocamium vulgare) à laquelle commence à s'ajouter la faune sessile: Chondrosiphon, Aglaophenia, etc. notons également la présence d" "Aglaozonia melanoidea» et nommons cette phase (bien qu'elle soit ici un peu accentuée par la saison) «phase à Valonia utricularis».

- Te peuplement n'est alors plus très éloigné du peuplement de référence (Fig. 4 et 6); la suite će l'évolution va voir Petroglossum nicaeense et Plocamium vulgare remplacer plus ou moins complètement, dans l'occupation du substrat, la plupart des autres espèces. Détail important: Petroglossum nicaeense et Plocamium vulgare ont encore, à ce moment là, des distributions relativement aléatoires.

\section{INFLUENCE DE L'EPOQUE DE LA DENUDATION}

Les relevés étudiés jusqu’ici (face Nord de lîle Riou) correspondent à une dénudation effectuée entre octobre et janvier; seul le relevé 336 (4ème mois) correspond à une dénudation estivale; nous avons néanmoins pu l'intégrer par la suite sans mal au Tableau 1: ses caractéristiques sont en effet, pour l'essentiel, intermédiaires entre celles des relevés 355 (3ème mois) et 333 (6ème mois). Ceci se voit très bien en particulier sur la Figure 6, où la constellation qui le représente ne s'intercalle pas trop mal entre celles des 3 et 6ème mois.

Pour nous faire une idée plus précise de l'influence de l'époque de la dénudation sur la régénération, nous avons comparé l'état de deux peuplements régénérés âgés de six mois; pour le premier (relevé 333, Tableau 1), la roche a été dénudée en janvier; pour le second (relevé 356 , ci-dessous), la roche a été dénudée au mois de juillet. Relevé $N^{\circ}$ 356. Face Nord de l'île Riou, près de Marseille. Dénudation le 3. 7. 1969. Prélèvement le 20.1.1970.

Espèces sciaphiles superficielles battues (SSBsI):

5.5 Dermatolithon hapalidioides var. confinis

2.3e Antithamnion elegans

1.4 Valonia utricularis

1.2j Petroglossum nicaeense

+e Acrochatium leptonema

$+\mathrm{j}$ Plocamium vulgare

$+\mathrm{j}$ Myriogramme minuta 
$+\mathrm{j}$ Aglaothamnion caudatum

tej Epilithon membranaceum

Espèces sciaphiles au sens large (SI + SIC):

1.4 Pleonosporium borreri

1.2 Apoglossum ruscifolium

1.2e Pseudochlorodesmis furcellata

Espèce infralittorale de substrat rocheux (ISR):

$+\quad$ Falkenbergia rufolanosa

Espèce photophile infralittorale (APsl):

1.2j Bryopsis duplex

Espèces sans signification précisée et espèces à large répartition écologique $(\mathrm{SSP}+\mathrm{LRE})$ :

1.1e Diatomées indéterminées

+ Cyanophytes indéterminés

+ Cladopborasp.

$+\mathrm{j}$ Ulvasp.

+ Derbesia tenuissima

te Licmophorasp.

te Erytbrocladia irregularis

te Erythrotrichia sp.

$+\quad$ Mélobésiées indéterminées

L'examen du relevé ci-dessus montre qu'il diffère assez sensiblement du relevé 333. En fait, il ne s'éloigne que très peu de la régénération suivie précédemment dans le Tableau 1 , à cette diffèrence près qu'il présente un important retard que nous pouvons chiffrer à deux ou trois mois.

Presque tous les paramètres du peuplement accusent en effet un retard de cet ordre: coefficient de similitude de KurczYnski avec le peuplement de référence (0.148); biomasse totale $\left(199 \mathrm{~g} / \mathrm{m}^{2}\right)$; nombre d'espèces dans le relevé (23); indice de diversité de MARgaLef (1.616).

Le coefficient de similitude avec de relevé 355 (3ème mois) et de 0.227 ; il est de 0.850 avec le relevé 336 (4ème mois) et de 0.496 avec le relevé 333 (6ème mois). C'est donc bien un retard de phase de deux mois qu'accuse le relevé âge de 6 mois dénudé en été par rapport à celui du même âge dénudé en hiver, puisque sa similitude maximale est réalisée avec le relevé 336 (4ème mois). Il semble donc (au moins à ce stade de la régénération) que la date de la dénudation n'influe pas sur la succession des phases, mais seulement sur leur durée.

\section{REGENERATION D'UN «FACIES MARGINAL» A CORALLINA MEDITERRANEA}

Il est évident que la structure de la biocoenose, et par conséquent le processus de sa régénération, ne correspondent pas à un modèle unique: les différents faciès, carac- 
térisés en particulier par l'exubérance d'une espèce ou d'une autre, peuvent présenter une structure assez originale (du moins en apparence) par rapport à celle du facièstype.

Nous avons donc étudié au Cap du Troc la structure d'un faciès à Corallina mediterranea, l'un des plus différents du faciès-type de ce point de vue.

Sans entrer dans le détail, le peuplement de référence présente ici quatre principaux éléments structuraux. Le premier est l'élément à Corallina mediterranea; il comporte en particulier Lomentaria articulata, Ceramium diapbanum, et quelques Delessériacées; cet élément, qui repose sur un fort concrétionnement de base, apparaît nettement en relief. Le second élément, très riche en espèces, est constitué par Plocamium vulgare, Petroglossum nicaeense et par des Mélobésiées encroûtantes. Le troisième élément est au contraire assez pauvre en espèces; il est surtout constitué par des touffes compactes de Cladophora pellucida. Le dernier enfin correspond notamment à des thalles de Mesophyllum licbenoides avec en épiphytes Halicystis paroula et Cutleria adspersa.

On a l'impression qu'ici l'élément le plus évolué (mais aussi le plus vulnérable à l'hydrodynamisme en raison de son relief et de son concrétionnement basal) est celui à Corallina mediterranea. On peut comparer cette «surévolution» au phénomène de brûlure de récif (reef-burn); il y a alors diminution de l'indice de diversité de MarGALEF et diminution générale du nombre d'espèces.

Les phénomènes de régénération sont ici compliqués par le grand nombre d'espèces (une cinquantaine) dans le peuplement de référence, et par le fait que certaines phases semblent facultatives: elles dépendent de l'époque de la dénudation. C'est ainsi qu'au printemps, par exemple, Corallina mediterranea peut «court-circuiter" la phase à Dermatolitbon bapalidioides en se réinstallant immédiatement sur le substrat dénudé.

Ceci mis à part, les conclusions générales que l'on peut tirer de l'étude de Riou restent valables, en particulier la rapidité de la régénération. Au 4ème mois (et que la dénudation ait lieu en janvier ou en mai), il y a déjà 40 à 43 espèces présentes sur la surface où est étudiée la régénération, la biomasse atteint 270 à $580 \mathrm{~g} / \mathrm{m}^{2}$, la densité de reproduction dépasse 2 (ce qui n'est plus très éloigné de la valeur de référence).

\section{CONCLUSIONS}

Comme l'étude structurale l'avait laissé supposer, les éléments structuraux mis en évidence semblent se retrouver, à peu de choses près, sous forme de phases au cours de la régénération du peuplement.

Ceci, joint au fait que Petroglossum nicaeense et Plocamium vulgare ont une distribution relativement aléatoire tout au long de la régénération alors qu'elle paraît contagieuse au niveau de peuplement de référence, conduit à penser que le peuplement évolue naturellement vers une phase à Petroglossum et Plocamium (au moins dans l'exemple choisi) assez vulnérable à l'hydrodynamisme, en particulier à cause de la taille de ces algues et du concrétionnement de base. Des touffes seraient alors arrachées par les vagues, principalement lors des tempêtes d'hiver.

L'assemblage en mosaïque de structures itératives, au niveau de peuplement de 
référence, serait donc dô à la présence simultanée, à quelques $\mathrm{cm}$ ou dizaines de $\mathrm{cm}$ de distance, de stades de régénération d'âge variable.

Par ailleurs, l'étude de la régénération permet de mettre en évidence la remarquable homéostasie de système; le véritable circuit «feed-back «, qui ramène la biocoenose RSB à son état moyen après une altération accidentelle ou provoquée, est probablement le plus efficace et le plus rapide de toutes les biocoenoses de substrat rocheux en Méditerranée (bien que les renseignements sur ce sujet soient encore assez fragmentaires); il l'est certainement en ce qui concerne les biocoenoses sciaphiles infralittorales et circalittorales.

Après un an, l'emplacement de la surface sur laquelle est étudiée la régénération est assez difficile à distinguer, sans analyse détaillée, du peuplement de référence, si ce n'est par l'absence de l'élément à Petroglossum nicaeense et Plocamium vulgare (ces deux espèces sont certes présentes, mais elles sont dispersées et ne constituent pas un «élément» au sens structural de terme).

Dans les conditions naturelles, cette régénération est sans doute encore plus rapide, puisque l'arrachage par les vagues est certainement moins efficace que celui auquel nous avons procédé, en cassant la roche sur quelques $\mathrm{mm}$ d'épaisseur, et que, d'autre part, les touffes arrachées sont de taille plus réduite que nos placettes de régénération, ce qui facilite la recolonisation par contagion latérale (bourgeonnement, stolons, etc.).

La production primaire, au cours de la première année de régénération, est donc supérieure à $1050 \mathrm{~g} / \mathrm{m}^{2}$ (poids humide essoré, «standing crop») pour le premier exemple étudié (la faune herbivore est peu abondante à ce moment-là mais ne doit toutefois pas être négligée), ce qui est une valeur très élevée, compte tenu de la biomasse bien modeste de la biocoenose-type RSB.

Pour le moment, l'hypothèse de l'évolution polycyclique (présence simultanée, en mosaïque, d'un grand nombre de cycles évoluant de façon parallèle, mais décalés les uns par rapport aux autres) repose plus sur des déductions floristico-statistiques que sur des observations réelles.

La seule indication en ce sens dont nous disposions est, à notre connaissance, celle de Bellan-Santint (1962), qui envisage que des thalles des Petroglossum nicaeense aient pu être arrachés lors de très grosses tempêtes d'hiver pour expliquer une diminution aberrante de la longueur moyenne des thalles. Mais il n'est pas impossible que cette diminution de la longueur des thalles trouve son explication dans la fertilité de l'algue, la partie centrale du thalle devenant alors moins résistante à la déchirure.

Pour notre part, nous devons reconnaître que nous n'avons que rarement observé des cicatrices certaines (car très rècentes) dues à l'arrachage par les vagues d'une portion du peuplement; peut-être la rapidité de la régénération en est elle la cause. Il reste donc à vérifier cette hypothèse par l'observation continue d'un peuplement convenablement repéré.

\section{RESUME}

1. La destruction expérimentale d'un peuplement rapporté à la biocoenose de la roche sciaphile en mode battu (RSB), dans la région de Marseille, et l'étude des phases 
de sa réinstallation (fraction algale), selon la méthode phytosociologique de BraunBLANQueT (1964), a apporté les résultats suivants.

2. Quatre mois après sa dénudation, la roche est déjà couverte par l'algue encroûtante Dermatolithon hapalidioides; ultérieurement (6 mois), de nombreuses algues dressées s'installent sur Dermatolitbon hapalidioides, principalement Pseudochlorodesmis furcellata, Petroglossum nicaeense et Myriogramme minuta. Au bout d'un an, toute la place disponible est occupée par Petroglossum nicaeense, Plocamium vulgare et Valonia utricularis, tandis que Dermatolitbon bapalidioides régresse an sous-strate. Ensuite, Petroglossum nicaeense et Plocamium vulgare deviennent codominantes.

3. Chacune de ces phases correspond à l'un des éléments structuraux du peuplement de référence. D'où l'hypothèse: le peuplement étudié évolue naturellement vers une phase à Petroglossum nicaeense et Plocamium vulgare assez vulnérable à l'hydrodynamisme; des touffes d'algues seraient arrachées par les vagues; l'assemblage en mosaïque d'éléments, au niveau de peuplement initial, serait donc dú à la présence simultanée, à quelques $\mathrm{cm}$ de distance, de stades de régénération d'âge variable.

4. La réinstallation du peuplement après sa destruction est excessivement rapide, et ceci pour un très grand nombre de paramètres envisagés.

\section{LITTERATURE CITEE}

Bellan-SantinI, D., 1962. Etude floristique et faunistique de quelques peuplementes infralittoraux de substrat rocheux. Recl. Trav. Stn. mar. Endoume 26 (41), 237-298.

- 1969. Contribution à l'étude des peuplements infralittoraux sur substrat rocheux (étude qualitative et quantitative de la frange supérieure). Recl. Trav. Stn. mar. Endoume 47 (63), $1-294$.

Boudouresque, C. F., 1969. Note préliminaire sur le peuplement algal des biotopes sciaphiles superficiels le long des côtes de l’Algérois et de la Kabylie. Bull. Mus. Hist. nat. Marseille 29, 165-187.

- 1971a. Contribution à l'étude phytosociologique des peuplements algaux des côtes varoises. Vegetatio 22, 83-184.

- 1971b. Sur les phytocoenoses sciaphiles superficialles en Méditerranée occidentale. Rapp. Commn int. Mer Médit. 20 (3), 193-196.

- 1971c. Méthodes d'étude qualitative et quantitative du benthos (en particulier du phytobenthos). Téthys 3 (1), 79-104.

- 1971d. Délimitation et signification des faciès algaux de la «Biocoenose de la Roche Sciaphile en mode Battu» dans la région de Marseille, par l'analyse factorielle des corrélations interspécifiques. Bull. Soc. phycol. Fr. 16, 31-37.

- \& LǘcK, H. B., 1972. Recherches de bionomie structurale au niveau d'un peuplement benthique sciaphile. J. exp. mar. Biol. Ecol. 8 (2), 133-144.

- \& Passelaigue, F., 1972. Note préliminaire sur le peuplement algal des biotopes sciaphiles superficiels battus dans la région de Marseille. Bull. Mus. Hist. nat. Marseille 32, 125-139.

Braun-Blanquet, J., 1959. Grundfragen und Aufgaben der Pflanzensoziologie. Pergamon Press, London, 145-171.

- 1964. Pflanzensoziologie. Springer, Wien, $865 \mathrm{pp}$.

Gounot, M., 1969. Méthodes d'étude quantitative de la végétation. Masson, Paris, 320 pp.

Huvk, P., 1969. Le stade initial et son importance réelle dans le peuplement algal des surfaces rocheuses du littoral marin. Proc. int. Seaweed Symp. 6, 201-211. 
- 1970. Recherches sur la genèse de quelques peuplements algaux marins de la roche littorale dans la région de Marseille. Thèse Doctorat Etat, Fac. Sci. Paris, $479 \mathrm{pp}$.

Kulczynskr, S., 1927. Die Pflanzenassoziationen des Pieninen (en polonais résumé allemand). Bull. int. Acad. pol. Sci. Lett. (B, Suppl.) 2, 57-203.

Peres, J.-M. \& Picard, J., 1964. Nouveau manuel de bionomie benthique de la mer Méditerranée. Recl. Trav. Stn. mar. Endoume 31 (47), 5-137.

L'adresse de l'auteur: Dr. C.-F. Boudouresque

Université d'Aix-Marseille

Laboratoire de Biologie Végétale Marine

Campus Universitaire de Luminy

Marseille- $9^{\circ}$

France 